\title{
ON THE GEOMETRY OF BIHARMONIC SUBMANIFOLDS IN SASAKIAN SPACE FORMS
}

\author{
D. FETCU AND C. ONICIUC
}

\begin{abstract}
We classify all proper-biharmonic Legendre curves in a Sasakian space form and point out some of their geometric properties. Then we provide a method for constructing anti-invariant proper-biharmonic submanifolds in Sasakian space forms. Finally, using the Boothby-Wang fibration, we determine all proper-biharmonic Hopf cylinders over homogeneous real hypersurfaces in complex projective spaces.
\end{abstract}

\section{INTRODUCTION}

As defined by Eells and Sampson in [14, harmonic maps $f:(M, g) \rightarrow(N, h)$ are the critical points of the energy functional

$$
E(f)=\frac{1}{2} \int_{M}\|d f\|^{2} v_{g}
$$

and they are solutions of the associated Euler-Lagrange equation

$$
\tau(f)=\operatorname{trace}_{g} \nabla d f=0,
$$

where $\tau(f)$ is called the tension field of $f$. When $f$ is an isometric immersion with mean curvature vector field $H$, then $\tau(f)=m H$ and $f$ is harmonic if and only if it is minimal.

The bienergy functional (proposed also by Eells and Sampson in 1964, 14) is defined by

$$
E_{2}(f)=\frac{1}{2} \int_{M}\|\tau(f)\|^{2} v_{g}
$$

The critical points of $E_{2}$ are called biharmonic maps and they are solutions of the Euler-Lagrange equation (derived by Jiang in 1986, 20]):

$$
\tau_{2}(f)=-\Delta^{f} \tau(f)-\operatorname{trace}_{g} R^{N}(d f, \tau(f)) d f=0,
$$

where $\Delta^{f}$ is the Laplacian on sections of $f^{-1} T N$ and $R^{N}(X, Y)=\nabla_{X} \nabla_{Y}-$ $\nabla_{Y} \nabla_{X}-\nabla_{[X, Y]}$ is the curvature operator on $N ; \tau_{2}(f)$ is called the bitension field of $f$. Since all harmonic maps are biharmonic, we are interested in studying those which are biharmonic but non-harmonic, called proper-biharmonic maps.

Now, if $f: M \rightarrow N_{c}$ is an isometric immersion into a space form of constant sectional curvature $c$, then

$$
\tau(f)=m H \quad \text { and } \quad \tau_{2}(f)=-m \Delta^{f} H+c m^{2} H .
$$

Thus $f$ is biharmonic if and only if

$$
\Delta^{f} H=m c H .
$$

Contribution to the Proceedings of the 10-th International Conference on Geometry, Integrability and Quantization, Varna 2008, Bulgaria. 
In a different way, Chen defined the biharmonic submanifolds in an Euclidean space as those with harmonic mean curvature vector field ([10]). Replacing $c=0$ in the above equation we just reobtain Chen's definition. Moreover, let $f: M \rightarrow \mathbb{R}^{n}$ be an isometric immersion. Set $f=\left(f^{1}, \ldots, f^{n}\right)$ and $H=\left(H^{1}, \ldots, H^{n}\right)$. Then $\Delta^{f} H=\left(\Delta H^{1}, \ldots, \Delta H^{n}\right)$, where $\Delta$ is the Beltrami-Laplace operator on $M$, and $f$ is biharmonic if and only if

$$
\Delta^{f} H=\Delta\left(\frac{-\Delta f}{m}\right)=-\frac{1}{m} \Delta^{2} f=0 .
$$

There are several classification results for the proper-biharmonic submanifolds in Euclidean spheres and non-existence results for such submanifolds in space forms $N_{c}, c \leq 0$ ([4], [5], 7], 8], [9], [10], [13]), while in spaces of non-constant sectional curvature only few results were obtained (11, [12, [18, [19, [25, [29]).

We recall that the proper-biharmonic curves of the unit Euclidean 2-dimensional sphere $\mathbb{S}^{2}$ are the circles of radius $\frac{1}{\sqrt{2}}$, and the proper-biharmonic curves of $\mathbb{S}^{3}$ are the geodesics of the minimal Clifford torus $\mathbb{S}^{1}\left(\frac{1}{\sqrt{2}}\right) \times \mathbb{S}^{1}\left(\frac{1}{\sqrt{2}}\right)$ with the slope different from \pm 1 . The proper-biharmonic curves of $\mathbb{S}^{3}$ are helices. Further, the properbiharmonic curves of $\mathbb{S}^{n}, n>3$, are those of $\mathbb{S}^{3}$ (up to a totally geodesic embedding). Concerning the hypersurfaces of $\mathbb{S}^{n}$, it was conjectured in [4] that the only properbiharmonic hypersurfaces are the open parts of $\mathbb{S}^{n-1}\left(\frac{1}{\sqrt{2}}\right)$ or $\mathbb{S}^{m_{1}}\left(\frac{1}{\sqrt{2}}\right) \times \mathbb{S}^{m_{2}}\left(\frac{1}{\sqrt{2}}\right)$ with $m_{1}+m_{2}=n-1$ and $m_{1} \neq m_{2}$.

Since odd dimensional unit Euclidean spheres $\mathbb{S}^{2 n+1}$ are Sasakian space forms with constant $\varphi$-sectional curvature 1, the next step is to study the biharmonic submanifolds of Sasakian space forms. In this paper we mainly gather the results obtained in [15, [16] and [17].

We note that the proper-biharmonic submanifolds in pseudo-Riemannian manifolds are also intensively-studied (for example, see [2], 3], [11]).

For a general account of biharmonic maps see 22 and The Bibliography of Biharmonic Maps [28.

Conventions. We work in the $C^{\infty}$ category, that means manifolds, metrics, connections and maps are smooth. The Lie algebra of the vector fields on $N$ is denoted by $C(T N)$.

\section{Sasakian Space Forms}

In this section we briefly recall some basic facts from the theory of Sasakian manifolds. For more details see [6].

A contact metric structure on a manifold $N^{2 n+1}$ is given by $(\varphi, \xi, \eta, g)$, where $\varphi$ is a tensor field of type $(1,1)$ on $N, \xi$ is a vector field on $N, \eta$ is an 1-form on $N$ and $g$ is a Riemannian metric, such that

$$
\left\{\begin{array}{c}
\varphi^{2}=-I+\eta \otimes \xi, \quad \eta(\xi)=1, \\
g(\varphi X, \varphi Y)=g(X, Y)-\eta(X) \eta(Y), \quad g(X, \varphi Y)=d \eta(X, Y),
\end{array}\right.
$$

for any $X, Y \in C(T N)$.

A contact metric structure $(\varphi, \xi, \eta, g)$ is Sasakian if it is normal, i.e.

$$
N_{\varphi}+2 d \eta \otimes \xi=0
$$

where 


$$
N_{\varphi}(X, Y)=[\varphi X, \varphi Y]-\varphi[\varphi X, Y]-\varphi[X, \varphi Y]+\varphi^{2}[X, Y], \quad \forall X, Y \in C(T N)
$$

is the Nijenhuis tensor field of $\varphi$.

The contact distribution of a Sasakian manifold $(N, \varphi, \xi, \eta, g)$ is defined by $\{X \in$ $T N: \eta(X)=0\}$, and an integral curve of the contact distribution is called Legendre curve.

A submanifold $M$ of $N$ which is tangent to $\xi$ is said to be anti-invariant if $\varphi$ maps any vector tangent to $M$ and normal to $\xi$ to a vector normal to $M$.

Let $(N, \varphi, \xi, \eta, g)$ be a Sasakian manifold. The sectional curvature of a 2-plane generated by $X$ and $\varphi X$, where $X$ is an unit vector orthogonal to $\xi$, is called $\varphi$-sectional curvature determined by $X$. A Sasakian manifold with constant $\varphi$ sectional curvature $c$ is called a Sasakian space form and it is denoted by $N(c)$.

A contact metric manifold $(N, \varphi, \xi, \eta, g)$ is called regular if for any point $p \in N$ there exists a cubic neighborhood of $p$ such that any integral curve of $\xi$ passes through the neighborhood at most once, and strictly regular if all integral curves are homeomorphic to each other.

Let $(N, \varphi, \xi, \eta, g)$ be a regular contact metric manifold. Then the orbit space $\bar{N}=$ $N / \xi$ has a natural manifold structure and, moreover, if $N$ is compact then $N$ is a principal circle bundle over $\bar{N}$ (the Boothby-Wang Theorem). In this case the fibration $\pi: N \rightarrow \bar{N}$ is called the Boothby-Wang fibration. The Hopf fibration $\pi: \mathbb{S}^{2 n+1} \rightarrow \mathbb{C} P^{n}$ is a well-known example of a Boothby-Wang fibration.

Theorem $2.1(24)$. Let $(N, \varphi, \xi, \eta, g)$ be a strictly regular Sasakian manifold. Then on $\bar{N}$ can be given the structure of a Kähler manifold. Moreover, if $(N, \varphi, \xi$, $\eta, g)$ is a Sasakian space form $N(c)$, then $\bar{N}$ has constant sectional holomorphic curvature $c+3$.

Even if $N$ is non-compact, we still call the fibration $\pi: N \rightarrow \bar{N}$ of a strictly regular Sasakian manifold, the Boothby-Wang fibration.

\section{Biharmonic Legendre Curves in Sasakian Space Forms}

Let $\left(N^{n}, g\right)$ be a Riemannian manifold and $\gamma: I \rightarrow N$ a curve parametrized by arc length. Then $\gamma$ is called a Frenet curve of osculating order $r, 1 \leq r \leq n$, if there exists orthonormal vector fields $E_{1}, E_{2}, \ldots, E_{r}$ along $\gamma$ such that $E_{1}=\gamma^{\prime}=T$, $\nabla_{T} E_{1}=\kappa_{1} E_{2}, \nabla_{T} E_{2}=-\kappa_{1} E_{1}+\kappa_{2} E_{3}, \ldots, \nabla_{T} E_{r}=-\kappa_{r-1} E_{r-1}$, where $\kappa_{1}, \ldots, \kappa_{r-1}$ are positive functions on $I$.

A geodesic is a Frenet curve of osculating order 1; a circle is a Frenet curve of osculating order 2 with $\kappa_{1}=$ constant; a helix of order $r, r \geq 3$, is a Frenet curve of osculating order $r$ with $\kappa_{1}, \ldots, \kappa_{r-1}$ constants; a helix of order 3 is called, simply, helix.

In [16] we studied the biharmonicity of Legendre Frenet curves and we obtained the following results.

Let $\left(N^{2 n+1}, \varphi, \xi, \eta, g\right)$ be a Sasakian space form with constant $\varphi$-sectional curvature $c$ and $\gamma: I \rightarrow N$ a Legendre Frenet curve of osculating order $r$. Then $\gamma$ is 
biharmonic if and only if

$$
\begin{aligned}
\tau_{2}(\gamma)= & \nabla_{T}^{3} T-R\left(T, \nabla_{T} T\right) T \\
= & \left(-3 \kappa_{1} \kappa_{1}^{\prime}\right) E_{1}+\left(\kappa_{1}^{\prime \prime}-\kappa_{1}^{3}-\kappa_{1} \kappa_{2}^{2}+\frac{(c+3) \kappa_{1}}{4}\right) E_{2} \\
& +\left(2 \kappa_{1}^{\prime} \kappa_{2}+\kappa_{1} \kappa_{2}^{\prime}\right) E_{3}+\kappa_{1} \kappa_{2} \kappa_{3} E_{4}+\frac{3(c-1) \kappa_{1}}{4} g\left(E_{2}, \varphi T\right) \varphi T \\
= & 0 .
\end{aligned}
$$

The expression of the bitension field $\tau_{2}(\gamma)$ imposed a case-by-case analysis as follows.

Case I $(c=1)$

Theorem 3.1 ([16]). If $c=1$ then $\gamma$ is proper-biharmonic if and only if $n \geq 2$ and either $\gamma$ is a circle with $\kappa_{1}=1$ or $\gamma$ is a helix with $\kappa_{1}^{2}+\kappa_{2}^{2}=1$.

Case II $\left(c \neq 1\right.$ and $\left.E_{2} \perp \varphi T\right)$

Theorem $3.2([16])$. Assume that $c \neq 1$ and $E_{2} \perp \varphi T$. We have

1) if $c \leq-3$ then $\gamma$ is biharmonic if and only if it is a geodesic;

2) if $c>-3$ then $\gamma$ is proper-biharmonic if and only if either

a) $n \geq 2$ and $\gamma$ is a circle with $\kappa_{1}^{2}=\frac{c+3}{4}$, or

b) $n \geq 3$ and $\gamma$ is a helix with $\kappa_{1}^{2}+\kappa_{2}^{2}=\frac{c+3}{4}$.

Case III $\left(c \neq 1\right.$ and $\left.E_{2} \| \varphi T\right)$

Theorem 3.3 ([16]). If $c \neq 1$ and $E_{2} \| \varphi T$, then $\{T, \varphi T, \xi\}$ is the Frenet frame field of $\gamma$ and we have

1) if $c<1$ then $\gamma$ is biharmonic if and only if it is a geodesic;

2) if $c>1$ then $\gamma$ is proper-biharmonic if and only if it is a helix with $\kappa_{1}^{2}=c-1$ (and $\kappa_{2}=1$ ).

Remark 3.4. In dimension 3 the result was obtained by Inoguchi in [19] and explicit examples are given in [15].

Case IV $\left(c \neq 1\right.$ and $g\left(E_{2}, \varphi T\right)$ is not constant 0,1 or -1$)$

Theorem $3.5([16])$. Let $c \neq 1$ and $\gamma$ a Legendre Frenet curve of osculating order $r$ such that $g\left(E_{2}, \varphi T\right)$ is not constant 0,1 or -1 . We have

1) if $c \leq-3$ then $\gamma$ is biharmonic if and only if it is a geodesic;

2) if $c>-3$ then $\gamma$ is proper-biharmonic if and only if $r \geq 4, \varphi T=\cos \alpha_{0} E_{2}+$ $\sin \alpha_{0} E_{4}$ and

$$
\left\{\begin{array}{l}
\kappa_{1}, \kappa_{2}, \kappa_{3}=\text { constant }>0 \\
\kappa_{1}^{2}+\kappa_{2}^{2}=\frac{c+3}{4}+\frac{3(c-1)}{4} \cos ^{2} \alpha_{0} \\
\kappa_{2} \kappa_{3}=-\frac{3(c-1)}{8} \sin \left(2 \alpha_{0}\right),
\end{array}\right.
$$

where $\alpha_{0} \in(0,2 \pi) \backslash\left\{\frac{\pi}{2}, \pi, \frac{3 \pi}{2}\right\}$ is a constant such that

$$
c+3+3(c-1) \cos ^{2} \alpha_{0}>0, \quad 3(c-1) \sin \left(2 \alpha_{0}\right)<0 .
$$


In order to obtain explicit examples of proper-biharmonic Legendre curves given by Theorem 3.1 we used the unit Euclidean sphere $\mathbb{S}^{2 n+1}$ as a model of a Sasakian space form with $c=1$ and we proved the following

Theorem 3.6 ([16]). Let $\gamma: I \rightarrow \mathbb{S}^{2 n+1}(1), n \geq 2$, be a proper-biharmonic Legendre curve parametrized by arc length. Then the parametric equation of $\gamma$ in the Euclidean space $\mathbb{E}^{2 n+2}=\left(\mathbb{R}^{2 n+2},\langle\rangle,\right)$ is either

$$
\gamma(s)=\frac{1}{\sqrt{2}} \cos (\sqrt{2} s) e_{1}+\frac{1}{\sqrt{2}} \sin (\sqrt{2} s) e_{2}+\frac{1}{\sqrt{2}} e_{3},
$$

where $\left\{e_{i}, \mathcal{I} e_{j}\right\}$ are constant unit vectors orthogonal to each other, or

$$
\gamma(s)=\frac{1}{\sqrt{2}} \cos (A s) e_{1}+\frac{1}{\sqrt{2}} \sin (A s) e_{2}+\frac{1}{\sqrt{2}} \cos (B s) e_{3}+\frac{1}{\sqrt{2}} \sin (B s) e_{4},
$$

where

$$
A=\sqrt{1+\kappa_{1}}, \quad B=\sqrt{1-\kappa_{1}}, \quad \kappa_{1} \in(0,1),
$$

$\left\{e_{i}\right\}$ are constant unit vectors orthogonal to each other such that

$$
\begin{gathered}
\left\langle e_{1}, \mathcal{I} e_{3}\right\rangle=\left\langle e_{1}, \mathcal{I} e_{4}\right\rangle=\left\langle e_{2}, \mathcal{I} e_{3}\right\rangle=\left\langle e_{2}, \mathcal{I} e_{4}\right\rangle=0 \\
A\left\langle e_{1}, \mathcal{I} e_{2}\right\rangle+B\left\langle e_{3}, \mathcal{I} e_{4}\right\rangle=0
\end{gathered}
$$

and $\mathcal{I}$ is the usual complex structure on $\mathbb{R}^{2 n+2}$.

Remark 3.7. For the Cases II and III we also obtained the explicit equations of proper-biharmonic Legendre curves in odd dimensional spheres endowed with the deformed Sasakian structure introduced in [27.

In 21] are introduced the complex torsions for a Frenet curve in a complex manifold. In the same way, for $\gamma: I \rightarrow N$ a Legendre Frenet curve of osculating order $r$ in a Sasakian manifold $\left(N^{2 n+1}, \varphi, \xi, \eta, g\right)$, we define the $\varphi$-torsions $\tau_{i j}=$ $g\left(E_{i}, \varphi E_{j}\right)=-g\left(\varphi E_{i}, E_{j}\right), i, j=1, \ldots, r, i<j$.

It is easy to see that

Proposition 3.8. Let $\gamma: I \rightarrow N(c)$ be a proper-biharmonic Legendre Frenet curve in a Sasakian space form $N(c), c \neq 1$. Then $c>-3$ and $\tau_{12}$ is constant.

Moreover

Proposition 3.9. If $\gamma$ is a proper-biharmonic Legendre Frenet curve in a Sasakian space form $N(c), c>-3, c \neq 1$, of osculating order $r<4$, then it is a circle or a helix with constant $\varphi$-torsions.

Proof. From Theorems 3.2, 3.3 and 3.5 we see that if $\gamma$ is a proper-biharmonic Legendre Frenet curve of osculating order $r<4$, then $\tau_{12}=0$ or $\tau_{12}= \pm 1$ and, obviously, we only have to prove that when $\gamma$ is a helix then $\tau_{13}$ and $\tau_{23}$ are constants.

Indeed, by using the Frenet equations of $\gamma$, we have

$$
\begin{aligned}
\tau_{13} & =g\left(E_{1}, \varphi E_{3}\right)=-\frac{1}{\kappa_{2}} g\left(\varphi E_{1}, \nabla_{E_{1}} E_{2}+\kappa_{1} E_{1}\right)=-\frac{1}{\kappa_{2}} g\left(\varphi E_{1}, \nabla_{E_{1}} E_{2}\right) \\
& =\frac{1}{\kappa_{2}} g\left(E_{2}, \nabla_{E_{1}} \varphi E_{1}\right)=\frac{1}{\kappa_{2}} g\left(E_{2}, \varphi \nabla_{E_{1}} E_{1}+\xi\right)=0
\end{aligned}
$$


since

$$
g\left(E_{2}, \xi\right)=\frac{1}{\kappa_{1}} g\left(\nabla_{E_{1}} E_{1}, \xi\right)=-\frac{1}{\kappa_{1}} g\left(E_{1}, \nabla_{E_{1}} \xi\right)=\frac{1}{\kappa_{1}} g\left(E_{1}, \varphi E_{1}\right)=0 .
$$

On the other hand, it is easy to see that for any Frenet curve of osculating order 3 we have $\tau_{23}=\frac{1}{\kappa_{1}}\left(\tau_{13}^{\prime}+\kappa_{2} \tau_{12}+\eta\left(E_{3}\right)\right)$ and

$$
\begin{aligned}
\eta\left(E_{3}\right) & =g\left(E_{3}, \xi\right)=\frac{1}{\kappa_{2}}\left(g\left(\nabla_{E_{1}} E_{2}, \xi\right)+\kappa_{1} g\left(E_{1}, \xi\right)\right)=-\frac{1}{\kappa_{2}} g\left(E_{2}, \nabla_{E_{1}} \xi\right) \\
& =-\frac{1}{\kappa_{2}} \tau_{12} .
\end{aligned}
$$

In conclusion, $\tau_{23}=\frac{1}{\kappa_{1}}\left(\tau_{13}^{\prime}+\kappa_{2} \tau_{12}-\frac{1}{\kappa_{2}} \tau_{12}\right)=$ constant.

Proposition 3.10. If $\gamma$ is a proper-biharmonic Legendre Frenet curve in a Sasakian space form $N(c)$ of osculating order $r=4$, then $c \in\left(\frac{7}{3}, 5\right)$ and the curvatures of $\gamma$ are

$$
\kappa_{1}=\frac{\sqrt{c+3}}{2}, \quad \kappa_{2}=\frac{1}{2} \sqrt{\frac{6(c-1)(5-c)}{c+3}}, \quad \kappa_{3}=\frac{1}{2} \sqrt{\frac{3(c-1)(3 c-7)}{c+3}} .
$$

Moreover, the $\varphi$-torsions of $\gamma$ are given by

$$
\left\{\begin{array}{ccc}
\tau_{12}=\mp \sqrt{\frac{2(5-c)}{c+3}}, & \tau_{13}=0, & \tau_{14}= \pm \sqrt{\frac{3 c-7}{c+3}} \\
\tau_{23}=\mp \frac{3 c-7}{\sqrt{3(c-1)(c+3)}}, & \tau_{24}=0, & \tau_{34}= \pm \sqrt{\frac{2(5-c)(3 c-7)}{3(c-1)(c+3)}} .
\end{array}\right.
$$

Proof. Let $\gamma$ be a proper-biharmonic Legendre Frenet curve in $N(c)$ of osculating order $r=4$. Then $c \neq 1$ and $\tau_{12}$ is different from 0,1 or -1 . From Theorem 3.5 we have $\varphi E_{1}=\cos \alpha_{0} E_{2}+\sin \alpha_{0} E_{4}$. It results that

$$
\tau_{12}=-\cos \alpha_{0}, \quad \tau_{13}=0, \quad \tau_{14}=-\sin \alpha_{0}, \quad \text { and } \quad \tau_{24}=0
$$

In order to prove that $\tau_{23}$ is constant we differentiate the expression of $\varphi E_{1}$ along $\gamma$ and using the Frenet equations we obtain

$$
\begin{aligned}
\nabla_{E_{1}} \varphi E_{1} & =\cos \alpha_{0} \nabla_{E_{1}} E_{2}+\sin \alpha_{0} \nabla_{E_{1}} E_{4} \\
& =-\kappa_{1} \cos \alpha_{0} E_{1}+\left(\kappa_{2} \cos \alpha_{0}-\kappa_{3} \sin \alpha_{0}\right) E_{3} .
\end{aligned}
$$

On the other hand, $\nabla_{E_{1}} \varphi E_{1}=\kappa_{1} \varphi E_{2}+\xi$ and therefore we have

$$
\kappa_{1} \varphi E_{2}+\xi=-\kappa_{1} \cos \alpha_{0} E_{1}+\left(\kappa_{2} \cos \alpha_{0}-\kappa_{3} \sin \alpha_{0}\right) E_{3} .
$$

We take the scalar product in (3.1) with $\xi$ and obtain

$$
\left(\kappa_{2} \cos \alpha_{0}-\kappa_{3} \sin \alpha_{0}\right) \eta\left(E_{3}\right)=1 .
$$

In the same way as in the proof of Proposition 3.9 we get

$$
\begin{aligned}
\eta\left(E_{3}\right) & =g\left(E_{3}, \xi\right)=\frac{1}{\kappa_{2}}\left(g\left(\nabla_{E_{1}} E_{2}, \xi\right)+\kappa_{1} g\left(E_{1}, \xi\right)\right)=-\frac{1}{\kappa_{2}} g\left(E_{2}, \nabla_{E_{1}} \xi\right) \\
& =-\frac{1}{\kappa_{2}} \tau_{12}=\frac{\cos \alpha_{0}}{\kappa_{2}}
\end{aligned}
$$

and then, from (3.2),

$$
\kappa_{2} \sin \alpha_{0}=-\kappa_{3} \cos \alpha_{0}
$$


Therefore $\alpha_{0} \in\left(\frac{\pi}{2}, \pi\right) \cup\left(\frac{3 \pi}{2}, 2 \pi\right)$.

Next, from Theorem 3.5, we have

$$
\kappa_{1}^{2}=\frac{c+3}{4}, \quad \kappa_{2}^{2}=\frac{3(c-1)}{4} \cos ^{2} \alpha_{0}, \quad \kappa_{3}^{2}=\frac{3(c-1)}{4} \sin ^{2} \alpha_{0},
$$

and so $c$ must be greater than 1 .

Now, we take the scalar product in (3.1) with $E_{3}, \varphi E_{2}$ and $\varphi E_{4}$, respectively, and we get

$$
\begin{gathered}
\kappa_{1} \tau_{23}=-\left(\kappa_{2} \cos \alpha_{0}-\kappa_{3} \sin \alpha_{0}\right)+\eta\left(E_{3}\right)=-\frac{\kappa_{2}}{\cos \alpha_{0}}+\frac{\cos \alpha_{0}}{\kappa_{2}} \\
\kappa_{1} \sin ^{2} \alpha_{0}=-\left(\kappa_{2} \cos \alpha_{0}-\kappa_{3} \sin \alpha_{0}\right) \tau_{23}=-\frac{\kappa_{2}}{\cos \alpha_{0}} \tau_{23}
\end{gathered}
$$

$$
0=\kappa_{1} \cos \alpha_{0} \sin \alpha_{0}+\left(\kappa_{2} \cos \alpha_{0}-\kappa_{3} \sin \alpha_{0}\right) \tau_{34}=\kappa_{1} \cos \alpha_{0} \sin \alpha_{0}+\frac{\kappa_{2}}{\cos \alpha_{0}} \tau_{34}
$$

and then, equations (3.3) and (3.4) lead to

$$
\kappa_{1}^{2} \sin ^{2} \alpha_{0}=\frac{\kappa_{2}^{2}}{\cos ^{2} \alpha_{0}}-1
$$

We come to the conclusion $\sin ^{2} \alpha_{0}=\frac{3 c-7}{c+3}$, so $c \in\left(\frac{7}{3}, 5\right)$, and then we obtain the expressions of the curvatures and the $\varphi$-torsions.

Remark 3.11. The proper-biharmonic Legendre curves given by Theorem 3.6 (for the case $c=1$ ) have also constant $\varphi$-torsions.

\section{A Method To Obtain Biharmonic Submanifolds in a Sasakian Space FORM}

In [16] we gave a method to obtain proper-biharmonic anti-invariant submanifolds in a Sasakian space form from proper-biharmonic integral submanifolds.

Theorem 4.1 ([16]). Let $\left(N^{2 n+1}, \varphi, \xi, \eta, g\right)$ be a strictly regular Sasakian space form with constant $\varphi$-sectional curvature $c$ and let $i: M \rightarrow N$ be an $r$-dimensional integral submanifold of $N, 1 \leq r \leq n$. Consider

$$
F: \widetilde{M}=I \times M \rightarrow N, \quad F(t, p)=\phi_{t}(p)=\phi_{p}(t)
$$

where $I=\mathbb{S}^{1}$ or $I=\mathbb{R}$ and $\left\{\phi_{t}\right\}_{t \in I}$ is the flow of the vector field $\xi$. Then $F$ : $\left(\widetilde{M}, \widetilde{g}=d t^{2}+i^{*} g\right) \rightarrow N$ is a Riemannian immersion and it is proper-biharmonic if and only if $M$ is a proper-biharmonic submanifold of $N$.

The previous Theorem provides a classification result for proper-biharmonic surfaces in a Sasakian space form, which are invariant under the flow-action of $\xi$.

Theorem 4.2 ([16]). Let $M^{2}$ be a surface of $N^{2 n+1}(c)$ invariant under the flowaction of the characteristic vector field $\xi$. Then $M$ is proper-biharmonic if and only if, locally, it is given by $x(t, s)=\phi_{t}(\gamma(s))$, where $\gamma$ is a proper-biharmonic Legendre curve.

Also, using the standard Sasakian 3-structure on $\mathbb{S}^{7}$, by iteration, Theorem 4.1 leads to examples of 3-dimensional proper-biharmonic submanifolds of $\mathbb{S}^{7}$. 


\section{Biharmonic Hopf Cylinders in a Sasakian Space Form}

Let $\left(N^{2 n+1}, \varphi, \xi, \eta, g\right)$ be a strictly regular Sasakian manifold and $\overline{\mathbf{i}}: \bar{M} \rightarrow \bar{N}$ a submanifold of $\bar{N}$. Then $M=\pi^{-1}(\bar{M})$ is the Hopf cylinder over $\bar{M}$, where $\pi: N \rightarrow \bar{N}=N / \xi$ is the Boothby-Wang fibration.

In [19] the biharmonic Hopf cylinders in a 3-dimensional Sasakian space form are classified.

Theorem 5.1 ([19]). Let $S_{\bar{\gamma}}$ be a Hopf cylinder, where $\bar{\gamma}$ is a curve in the orbit space of $N^{3}(c)$, parametrized by arc length. We have

1) if $c \leqslant 1$, then $S_{\bar{\gamma}}$ is biharmonic if and only if it is minimal;

2) if $c>1$, then $S_{\bar{\gamma}}$ is proper-biharmonic if and only if the curvature $\bar{\kappa}$ of $\bar{\gamma}$ is constant $\bar{\kappa}^{2}=c-1$.

In 17. we obtained a geometric characterization of biharmonic Hopf cylinders of any codimension in an arbitrary Sasakian space form. A special case of our result is the case when $\bar{M}$ is a hypersurface.

Proposition 5.2 ([17]). If $\bar{M}$ is a hypersurface of $\bar{N}$, then $M=\pi^{-1}(\bar{M})$ is biharmonic if and only if

$$
\left\{\begin{array}{l}
\Delta^{\perp} H=\left(-\|B\|^{2}+\frac{c(n+1)+3 n-1}{2}\right) H \\
2 \operatorname{trace} A_{\nabla \perp H}(\cdot)+n \operatorname{grad}\left(\|H\|^{2}\right)=0,
\end{array}\right.
$$

where $B, A$ and $H$ are the second fundamental form of $M$ in $N$, the shape operator and the mean curvature vector field, respectively, and $\nabla^{\perp}$ and $\Delta^{\perp}$ are the normal connection and Laplacian on the normal bundle of $M$ in $N$.

Proposition 5.3 ([17]). If $\bar{M}$ is a hypersurface and $\|\bar{H}\|=$ constant $\neq 0$, then $M=\pi^{-1}(\bar{M})$ is proper-biharmonic if and only if

$$
\|\bar{B}\|^{2}=\frac{c(n+1)+3 n-5}{2} .
$$

Remark 5.4. From the last result we see that there exist no proper-biharmonic hypersurfaces of constant mean curvature $M=\pi^{-1}(\bar{M})$ in $N(c)$ if $c \leq \frac{5-3 n}{n+1}$, which implies that such hypersurfaces do not exist if $c \leq-3$, whatever the dimension of $N$ is.

From now on we shall consider $c>-3$.

In 26] Takagi classified all homogeneous real hypersurfaces in the complex projective space $\mathbb{C} P^{n}, n>1$, and found five types of such hypersurfaces (see also [23]). The first type (with subtypes $A 1$ and $A 2$ ) are described in the following.

We shall consider $u \in\left(0, \frac{\pi}{2}\right)$ and $r$ a positive constant given by $\frac{1}{r^{2}}=\frac{c+3}{4}$.

Theorem 5.5 ([26]). The geodesic spheres (Type A1) in complex projective space $\mathbb{C} P^{n}(c+3)$ have two distinct principal curvatures: $\lambda_{2}=\frac{1}{r} \cot u$ of multiplicity $2 n-2$ and $a=\frac{2}{r} \cot (2 u)$ of multiplicity 1 .

Theorem 5.6 (26]). The hypersurfaces of Type A2 in complex projective space $\mathbb{C} P^{n}(c+3)$ have three distinct principal curvatures: $\lambda_{1}=-\frac{1}{r} \tan u$ of multiplicity $2 p, \lambda_{2}=\frac{1}{r} \cot u$ of multiplicity $2 q$, and $a=\frac{2}{r} \cot (2 u)$ of multiplicity 1 , where $p>0$, $q>0$, and $p+q=n-1$. 
We note that if $c=1$ and $\bar{M}$ is of type $A 1$ or $A 2$ then $\pi^{-1}(\bar{M})=\mathbb{S}^{1}(\cos u) \times$ $\mathbb{S}^{2 n-1}(\sin u) \subset \mathbb{S}^{2 n+1}$ or $\pi^{-1}(\bar{M})=\mathbb{S}^{2 p+1}(\cos u) \times \mathbb{S}^{2 q+1}(\sin u)$, respectively.

By using Takagi's result we classified in [17] the biharmonic Hopf cylinders $M=$ $\pi^{-1}(\bar{M})$ in a Sasakian space form $N^{2 n+1}$ over homogeneous real hypersurfaces in $\mathbb{C} P^{n}, n>1$.

Theorem 5.7 ([17]). Let $M=\pi^{-1}(\bar{M})$ be the Hopf cylinder over $\bar{M}$.

1) If $\bar{M}$ is of Type A1, then $M$ is proper-biharmonic if and only if either

a) $c=1$ and $\tan ^{2} u=1$, or

b) $c \in\left[\frac{-3 n^{2}+2 n+1+8 \sqrt{2 n-1}}{n^{2}+2 n+5},+\infty\right) \backslash\{1\}$ and

$$
\begin{aligned}
\tan ^{2} u= & n+\frac{2 c-2}{c+3} \\
& \pm \frac{\sqrt{c^{2}\left(n^{2}+2 n+5\right)+2 c\left(3 n^{2}-2 n-1\right)+9 n^{2}-30 n+13}}{c+3} .
\end{aligned}
$$

2) If $\bar{M}$ is of Type A2, then $M$ is proper-biharmonic if and only if either

a) $c=1, \tan ^{2} u=1$ and $p \neq q$, or

b) $c \in\left[\frac{-3(p-q)^{2}-4 n+4+8 \sqrt{(2 p+1)(2 q+1)}}{(p-q)^{2}+4 n+4},+\infty\right) \backslash\{1\}$ and

$$
\begin{aligned}
\tan ^{2} u= & \frac{n}{2 p+1}+\frac{2 c-2}{(c+3)(2 p+1)} \\
& \pm \frac{\sqrt{c^{2}\left((p-q)^{2}+4 n+4\right)+2 c\left(3(p-q)^{2}+4 n-4\right)+9(p-q)^{2}-12 n+4}}{(c+3)(2 p+1)} .
\end{aligned}
$$

Theorem 5.8 ([17]). There are no proper-biharmonic hypersurfaces $M=\pi^{-1}(\bar{M})$ when $\bar{M}$ is a hypersurface of Type $B, C, D$ or $E$ in the complex projective space $\mathbb{C} P^{n}(c+3)$.

\section{ACKNOWLEDGEMENTS}

The authors were partially supported by the Grant CEEX, ET, 5871/2006 and by the Grant CEEX, ET, 5883/2006, Romania.

The first author would like to thank to the organizers, especially to Professor I. Mladenov, for the Conference Grant.

\section{REFERENCES}

[1] K. Arslan, R. Ezentas, C. Murathan and T. Sasahara, Biharmonic Anti-invariant Submanifolds in Sasakian Space Forms, Beitrge Algebra Geom., 48 (1)(2007), 191-207.

[2] A. Arvanitoyeorgos, F. Defever, G. Kaimakamis and V.J. Papantoniou, Biharmonic Lorentz Hypersurfaces in $E_{1}^{4}$, Pacific J. Math., 229 (2007), 293-305.

[3] A. Arvanitoyeorgos, F. Defever and G. Kaimakamis, Hypersurfaces of $E_{s}^{4}$ with Proper Mean Curvature Vector, J. Math. Soc. Japan, 59 (2007), 797-809.

[4] A. Balmus, S. Montaldo and C. Oniciuc, Classification Results for Biharmonic Submanifolds in Spheres, Israel J. Math., to appear.

[5] A. Balmuş, S. Montaldo and C. Oniciuc, Biharmonic Hypersurfaces in 4-Dimensional Space Forms, Math. Nachr., to appear.

[6] D.E. Blair, Riemannian Geometry of Contact and Symplectic Manifolds, Birkhäuser Boston, Progress in Mathematics, Volume 203, 2002.

[7] R. Caddeo, S. Montaldo and C. Oniciuc, Biharmonic Submanifolds of $\mathbb{S}^{3}$, Internat. J. Math., 12 (2001), 867-876.

[8] R. Caddeo, S. Montaldo and C. Oniciuc, Biharmonic Submanifolds in Spheres, Israel J. Math., 130 (2002), 109-123. 
[9] R. Caddeo, S. Montaldo and P. Piu, Biharmonic Curves on a Surface, Rend. Mat. Appl., (7) 21 (2001), 143-157.

[10] B.Y. Chen, A Report on Submanifolds of Finite Type, Soochow J. Math., 22 (1996), 117-337.

[11] B.Y. Chen, Classification of Marginally Trapped Lorentzian Flat Surfaces in $\mathbb{E}_{2}^{4}$ and Its Application to Biharmonic Surfaces, J. Math. Anal. Appl., 340 (2008), 861-875.

[12] J.T. Cho, J. Inoguchi and J.-E. Lee, Biharmonic Curves in 3-Dimensional Sasakian Space Form, Ann. Math. Pura Appl., 186 (2007), 685-701.

[13] I. Dimitric, Submanifolds of $\mathbb{E}^{m}$ with Harmonic Mean Curvature Vector, Bull. Inst. Math. Acad. Sinica, 20 (1992), 53-65.

[14] J. Eells and J.H. Sampson, Harmonic Mappings of Riemannian Manifolds, Amer. J. Math., 86 (1964), 109-160.

[15] D. Fetcu and C. Oniciuc, Explicit Formulas for Biharmonic Submanifolds in Non-Euclidean 3-Spheres, Abh. Math. Sem. Univ. Hamburg, 77 (2007), 179-190.

[16] D. Fetcu and C. Oniciuc, Explicit Formulas for Biharmonic Submanifolds in Sasakian Space Forms, arXiv:math.DG/0706.4160v1.

[17] D. Fetcu and C. Oniciuc, Biharmonic Hypersurfaces in Sasakian Space Forms, Preprint.

[18] T. Ichiyama, J. Inoguchi and H. Urakawa, Bi-harmonic Maps and Bi-Yang-Mills Fields, Note Mat., to appear.

[19] J. Inoguchi, Submanifolds with Harmonic Mean Curvature in Contact 3-manifolds, Colloq. Math., 100 (2004), 163-179.

[20] G.Y. Jiang, 2-harmonic Maps and Their First and Second Variational Formulas, Chinese Ann. Math. Ser. A, 7 (4)(1986), 389-402.

[21] S. Maeda and Y. Ohnita, Helical Geodesic Immersions into Complex Space Forms, Geom. Dedicata, 30 (1989), 93-114.

[22] S. Montaldo and C. Oniciuc, A Short Survey on Biharmonic Maps Between Riemannian Manifolds, Rev. Un. Mat. Argentina, 47 (2)(2006), 1-22.

[23] R. Niebergall and P.J. Ryan, Real Hypersurfaces in Complex Space Forms, Tight and Taut Submanifolds, MSRI Publications, 32 (1997), 233-305.

[24] K. Ogiue, On Fiberings of Almost Contact Manifolds, Kōdai Math. Sem. Rep., 17 (1965), $53-62$.

[25] T. Sasahara, Legendre Surfaces in Sasakian Space Forms Whose Mean Curvature Vectors Are Eigenvectors, Publ. Math. Debrecen, 67 (3-4)(2005), 285-303.

[26] R. Takagi, On Homogeneous Real Hypersurfaces in a Complex Projective Space, Osaka J. Math., 10 (1973), 495-506.

[27] S. Tanno, The Topology of Contact Riemannian Manifolds, Ill. J. Math., 12 (1968), 700-717.

[28] The Bibliography of Biharmonic Maps. http://beltrami.sc.unica.it/ biharmonic/.

[29] W. Zhang, New Examples of Biharmonic Submanifolds in $\mathbb{C} P^{n}$ and $\mathbb{S}^{2 n+1}$, arXiv:math.DG/07053961v1.

Department of Mathematics, "Gh. Asachi" Technical University of Iasi, Bd. Carol I NO. 11, 700506 IASI, ROMANIA

E-mail address: dfetcu@math.tuiasi.ro

Faculty of Mathematics, "Al.I. Cuza" University of Iasi, Bd. Carol I no. 11, 700506 IASI, ROMANIA

E-mail address: oniciucc@uaic.ro 